\title{
Electrical Stimulation in Treatment of Pharyngolaryngeal Dysfunctions
}

\author{
Simone Miller Michael Jungheim Daniela Kühn Martin Ptok \\ Department of Phoniatrics and Pediatric Audiology, Hanover Medical School, Hanover, Germany
}

\section{Key Words}

Neuromuscular electrical stimulation · Dysphonia .

Dysphagia $\cdot$ Dyspnoea $\cdot$ Laryngeal dysfunction

\begin{abstract}
Objective: Neuromuscular electrical stimulation (NMES) has been proposed in the treatment of laryngopharyngeal dysfunctions (dysphonia, dyspnoea, dysphagia) for more than 40 years. Several studies have investigated possible therapeutic effects. Some researchers described favourable results, whereas others did not find relevant benefits. This article aims to review available studies to give an overview regarding the current state of knowledge. Methods: We conducted a selective literature search using PubMed. Results: In total, 356 papers were identified: 6 case reports, 11 reviews, 43 prospective clinical trials and 3 retrospective trials were found. Conclusion: Due to different stimulation protocols, electrode positioning and various underlying pathological conditions, summarizing the present studies appears to be difficult. However, there is evidence that NMES is a valuable adjunct in patients with dysphagia and in patients with vocal fold paresis. Nevertheless, more empirical data is needed to fully understand the benefits provided by NMES. Further research suggestions are put forward.
\end{abstract}

\section{KARGER}

E-Mail karger@karger.com www.karger.com/fpl

\section{Introduction}

\section{Electrical Stimulation/Electric Currents}

The idea to use electric currents in a healing context is not new. Wall-paintings of the ancient Egyptians suggest that electricity from an electric catfish had already been used in ancient medicine [1]. Records of Scribonius Largus, a doctor in the Roman Empire, state that 'piscine electrotherapy', namely a live black torpedo, was used for pain treatment. It was said that this therapy cured one of Tiberius' freemen [2]. In the 19th century utilization of electrical energy in therapy re-emerged as an alternative to physiotherapy.

Today neuromuscular electrical stimulation (NMES) is well established in medicine. Even though its muscle strengthening effect in normal muscles is still under debate, there seems to be a lot of support regarding its effectiveness in strengthening weakened musculature, especially in combination with voluntary activity [3].

The term NMES is sometimes used to describe indirect muscle stimulation, where a muscle contraction is achieved due to stimulation of the innervating nerve [4]. In this article, however, we will use it generically [3] to describe stimulation of the nerve-muscle entity, i.e. direct [4] as well as indirect muscle stimulation. We will also include electric stimulation to modify sensibility if per- 
formed to enhance muscle activity, even though such stimulation could be classified as sensory stimulation.

In general three main frequency ranges of electric currents are distinguished. Currents with frequencies above $100,000 \mathrm{~Hz}$ have no stimulation effects and are used to warm the tissue in thermotherapy. In the low frequency range, up to $1 \mathrm{kHz}$, each pulse of sufficient intensity and duration can generate a muscle contraction [3]. In the so-called medium frequency range, exceeding $1 \mathrm{kHz}$, not all phases of currents can create a muscle contraction.

In paretic muscles electrostimulation aims to condition the muscle to prevent fibrosis and maintain flexibility until reinnervation occurs [5] and should be commenced as soon as possible. For denervated muscles, the best results seem to be achieved with vigorous isometric muscle contractions to the point of fatigue 2 to 3 times a day. All the denervated muscle fibres must be activated and isometric contractions are said to be more effective than isotonic [3].

\section{Larynx Functions and Swallowing}

The larynx represents an intersection between the airway and digestive tracts. It most importantly functions to protect the airways during swallowing and allows air passage during respiration, respectively. Furthermore it is the source of phonation [6].

Many articles investigated the effectiveness of NMES as a treatment for dysphagia. In this article we will review the benefit of NMES in the treatment of three laryngeal dysfunctions, such as dysphonia, dyspnoea and dysphagia, in order to give an overview regarding the current state of knowledge in these fields of research.

\section{Methods}

A literature search using PubMed according to current guidelines (www.prisma-statement.org) using the following key words was conducted: 'electric AND/OR electrical stimulation AND dysphonia OR dyspnea OR deglutition OR dysphagia OR laryngeal'.

The initial search resulted in a total of 356 hits. Only publications in English and German were included. After excluding multiple hits, articles with a different (topical) focus, implants or investigation on muscles other than the throat or neck or not in human adults, 6 case reports, 11 reviews, 43 prospective clinical trials, 3 retrospective trials and two meta-analyses were identified. (One meta-analysis was identified during the initial literature search. The second meta-analysis was published and included during the review process of this article.) Additional studies were identified from the references of the identified articles.

Electrical Stimulation for

Pharyngolaryngeal Dysfunction

\section{NMES for Dysphonia and Dyspnoea}

Dysphonia and dyspnoea may result from e.g. vocal fold paresis resulting from recurrent laryngeal nerve paresis, vocal fold bowing, spasmodic dysphonia and muscle tension dysphonia.

Apart from traditional voice therapy NMES alone or in combination with voice exercises has been used for more than 40 years [7-9].

\section{Vocal Fold Paresis}

Vocal fold pareses can be a sequela of central or peripheral nerve damage. Several studies investigated the effect of transcutaneous NMES on unilateral [10-12] and/or bilateral [13] vocal fold paresis, others tried more invasive forms of application, such as needle electrodes placed in the middle portion of the laryngeal abductor muscle [12]. Ptok and Strack $[10,11]$ and Dahl and Witt [13] compared the effectiveness of conventional voice therapy in comparison with electrical stimulation-supported voice exercise. Both groups found superior therapy results in the NMES groups regarding voice parameters, such as vocal fold irregularity $[10,11]$ and maximum phonation time $[10,11]$ or regeneration rates [13] and enhancement periods [13]. Zealear et al. [12] found that the magnitude of electrically induced vocal fold abduction during inspiration was comparable to the spontaneous movement on the unimpeded side.

\section{Vocal Fold Bowing}

In dysphonic patients laryngoscopy sometimes reveals vocal fold bowing either on one side or bilaterally. Mechanisms of vocal fold bowing are presumed but unknown $[14,15]$. It may stem from insufficient vocal fold muscle tension or degeneration of tissue in the vocal fold [14]. Treatment of vocal fold bowing usually involves laryngeal augmentation or thyroplastic medialization [14]. However, the effectiveness of voice therapy $[14,16]$, as well as behavioural voice therapy with adjunctive NMES delivered transcutaneously to the cricothyroid muscles and the superior laryngeal nerves has been suggested. LaGorio et al. [14] reported positive results regarding mean phonation times, voice handicap index values and glottal closure as a result of NMES. Mean phonation time for sustained phonation of the vowel [i:] increased significantly and post-stimulation voice handicap index values, relative to pre-stimulation values, tended towards significance. Also, glottal closure during phonation was said to improve and supraglottic compression decreased following stimulation. Improvements were still present or enhanced during a follow-up evaluation. 


\section{Spasmodic Dysphonia}

When suffering from spasmodic dysphonia the patients' voice sounds as if being strangled. The underlying pathomechanism is unclear. Ludlow et al. [17] suggest a central disinhibition of laryngeal responses to sensory input for adductor spasmodic dysphonia. Nowadays, the intralaryngeal injection of botulinum toxin (Botox) is the therapy of choice [18]. Several studies investigated the effects of NMES on adductor [17] as well as abductor [18] spasmodic dysphonia [19], all of which have used invasive electrodes, such as needle $[17,19]$ and hooked wire electrodes [18] applied to the region of the recurrent laryngeal nerve [19], superior laryngeal nerve [17], or the two laryngeal adductor muscle groups (thyroarytenoid muscles and lateral cricoarytenoid muscles) [18]. At least some studies suggested that NMES potentially improves voice quality in patients with spasmodic dysphonia $[18,19]$.

\section{Muscle Tension Dysphonia}

Muscle tension dysphonia is characterized by a change in voice quality and may result from a misuse of the voice. Organic factors resulting from such a misuse can involve nodules, phonation fissures, inflammation and oedema [20]. Therapeutic procedures usually involve voice therapy; however, surgical procedures might be necessary in certain cases. Guirro et al. [21] found that transcutaneous electrical nerve stimulation (a subtype of NMES) resulted in a decrease in hoarseness, the level of dysphonia and laryngeal injuries as well as relief of pain in women with nodules or bilateral mucus thickening and phonation fissure.

Other

Some studies also focused on the effects of NMES in healthy individuals $[22,23]$. Among others, differences in fundamental frequency and relative sound level (not significant [23]) were found.

\section{Dysphagia}

Swallowing is a vital mechanism and comprises the passage of foods and liquids as well as saliva from the oral cavity to the stomach. Aspects of the swallowing mechanism also serve to protect the lower respiratory tract from aspiration [24]. Four phases can be distinguished: anticipatory phase, oral phase, pharyngeal phase, and oesophageal phase.

Functional dysphagia therapy [25] is generally based on three components: restitution, compensation and adaptation. Restitution aims to fully or partially restore function and is based on stimulation (thermal, tactile), mobilization (pressure-resistance tasks) and motion exercise (labial, lingual exercise), to facilitate desired actions and inhibit unwanted movements. Compensatory strategies are chosen to improve remaining functions or replace swallowing dysfunctions. Swallowing manoeuvres (e.g. easy or effortful breath-holding, effortful swallow or Mendelsohn manoeuvre) or changes in posture (e.g. chin-tuck, head rotation) are commonly used to facilitate swallowing. Adaptation is concerned with adjusting the surrounding to the needs and abilities of the dysphagic patient by means of consistency modification of foods and liquids, adjuvants, and so forth.

\section{NMES and Dysphagia}

Studies using NMES to treat dysphagia seem to be incommensurable due to various electrodes and electrode placements, varying stimulation protocols, different aims regarding the outcome/achievements, varying study protocols and, most importantly, conflicting results from various studies.

\section{Electrodes and Electrode Placements}

For electrode placement the anterior neck can be divided into an upper submental region and a lower throat region. The upper region lies between the mandible and the hyoid bone, the lower region represents the area below the hyoid. Depending on the positioning of the electrodes and the current intensity, there is variation in how far the electrical current spreads in the tissue. The most superficial muscles will be reached first. With increasing intensity the current passes deeper into the tissue and stimulates deeper muscle layers [26]. Placement of electrodes in the upper region is thought to support laryngeal elevation during deglutition. Placing the electrodes below the hyoid can either help vocal fold closure or stimulate the antagonistic muscles which lower the larynx.

The platysma, in both regions, is the most superficial muscle, which as part of the facial muscles tightens the skin and is not involved in swallowing. In the submental region, the anterior belly of the digastric muscle lies directly underneath the platysma. It can raise the hyoid when teeth are occluded. The mylohyoid muscle and geniohyoid muscle (deeper than the mylohyoid) are situated underneath the digastric muscle. The mylohyoid muscle raises the hyoid upwards to the mandible; the geniohyoid muscle moves the hyoid in an upward and anterior direction. When stimulated, these muscles support elevation of the hyoid [26].

In the anterior neck region the first muscle beneath the platysma is the sternohyoid muscle. This muscle pulls the hyoid down towards the sternum. Underneath the ster- 
nohyoid lies the omohyoid muscle, which moves the hyoid downwards and laterally. Laryngeal elevation is only achieved by stimulating the thyrohyoid muscle, which is situated underneath these muscles [26].

The current may be applied directly into the muscle through bipolar needle electrodes [27], an intraluminal bipolar ring electrode catheter inside the pharynx [28], or transcutaneously by electrodes on the skin directly above the muscle [3].

\section{Stimulation Protocols}

Regarding studies in the field of NMES and dysphagia, implementation of a uniform stimulation protocol concerning the duration of each session, the total number of sessions or parameters of the electric currents has not been achieved.

Aims regarding the Outcome of NMES Application

Numerous studies have been undertaken during the initial - acute - phase after stroke. Therefore spontaneous remission cannot be ruled out as contributing to a pattern of results in a given study. In general the application of NMES in dysphagia therapy aims to improve laryngeal elevation (e.g. [26, 29]), improve glottal closure (e.g. [11, $14])$, improve/evoke reflex triggering (e.g. $[30,31])$, reduce aspiration (e.g. [32-35]), reduce spasticity (e.g. [36]), and increase quality of life (e.g. [37, 38])

\section{Study Protocols}

Published studies concerned with NMES and dysphagia can be roughly classified into two groups: those including patients and those including healthy volunteers. While the former investigate therapeutic effects, the latter use NMES to study the central/peripheral control mechanisms and pathways of swallowing.

\section{Conflicting Results}

Several studies have reported a positive effect of NMES in the treatment of dysphagia (e.g. [39-44]). Others [36, 45] suggested a negative effect of electric stimulation on hyolaryngeal elevation $[46,47]$ or found no significant differences between traditional therapeutic methods and NMES [48-53].

\section{Studies Evaluating NMES in Dysphagic Patients}

Many of the studies investigating the effect of NMES in the treatment of dysphagia compared swallowing ability and the occurrence of penetration and aspiration before and after therapy as indicators of the effectiveness of the therapeutic procedure $[29,32,34,37,39-44,48,49$,
51-60 amongst others, see tables 1, 3 for an overview]. Some studies, especially the earlier ones, were criticized regarding their study designs $[29,32,34]$. Freed et al. [34], for example, in their first evaluation of the VitalStim ${ }^{\circledR}$ device, did not elucidate specific treatment effects and no randomization was applied. Furthermore spontaneous regeneration was not controlled for. The number of therapeutic sessions between the two treatment conditions was found to be unequal.

Most studies used a transcutaneous form of current application, others [51] stimulated intraorally or used a catheter for pharyngeal simulation [61] (see table 1 for an overview regarding the different results and stimulation protocols of studies investigating NMES in dysphagic patients).

In 2007 a meta-analysis was conducted on 7 studies, including 255 patients with dysphagia of multiple aetiologies, to examine the effects of NMES in improving clinical swallowing ability. The analysis showed a statistically significant, summary effect size supporting the use of NMES in the rehabilitation of adult patients with dysphagia with a mean improvement of $20 \%$ in swallowing performance following treatment [62]. The authors stated themselves, however, that most of the studies included in the metaanalysis showed severe methodological flaws, such as unblinded observers, no randomization to conditions, or the absence of a control group. A more recent meta-analysis [38] investigating 7 studies comparing NMES versus traditional therapy suggests that in all patient groups, except for stroke patients, NMES was more effective than traditional therapy. In post-stroke dysphagia patients the effectiveness of NMES and traditional therapy were comparable. Similar results had previously been reported in a review by Clark et al. [63].

Numerous case reports on patients with various types of dysphagia also suggest great improvements in swallowing functions [64-69]. From the 25 studies [29, 32, 34, $37-44,48,49,51-62]$ identified in this literature search, 18 reported positive results for NMES treatment of dysphagia (table 1).

\section{Studies regarding the Influence of NMES on}

Swallowing Mechanisms in Healthy Subjects

Various studies investigated the influence of NMES on swallowing mechanisms in healthy subjects (see table 2 for an overview), such as laryngeal elevation [38], vocal fold closure [27, 70] and swallowing frequency [71]. Also different stimulation conditions were investigated, e.g. different electrode placements $[72,73]$, stimulation conditions [71] or stimulation currents [46]. 


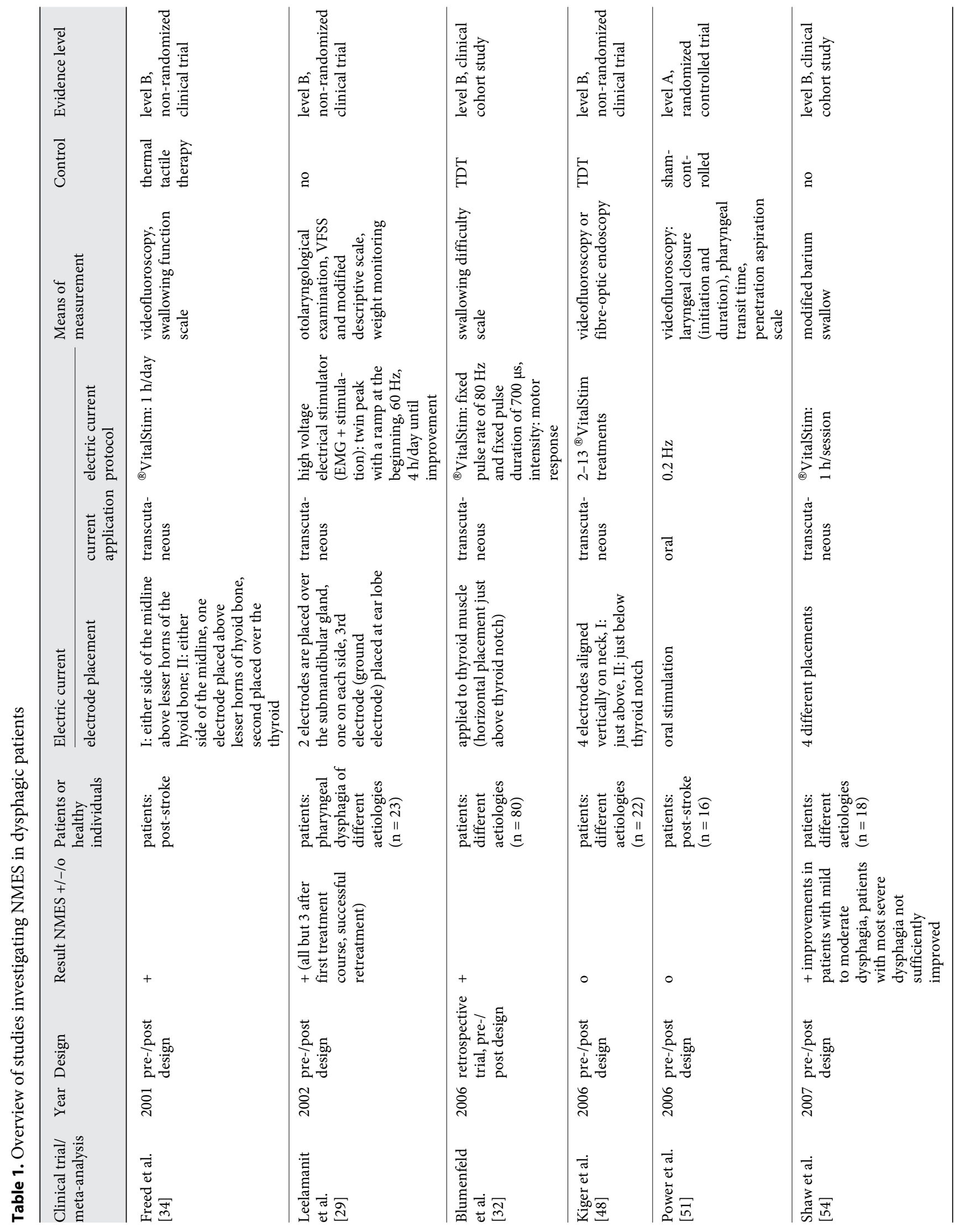




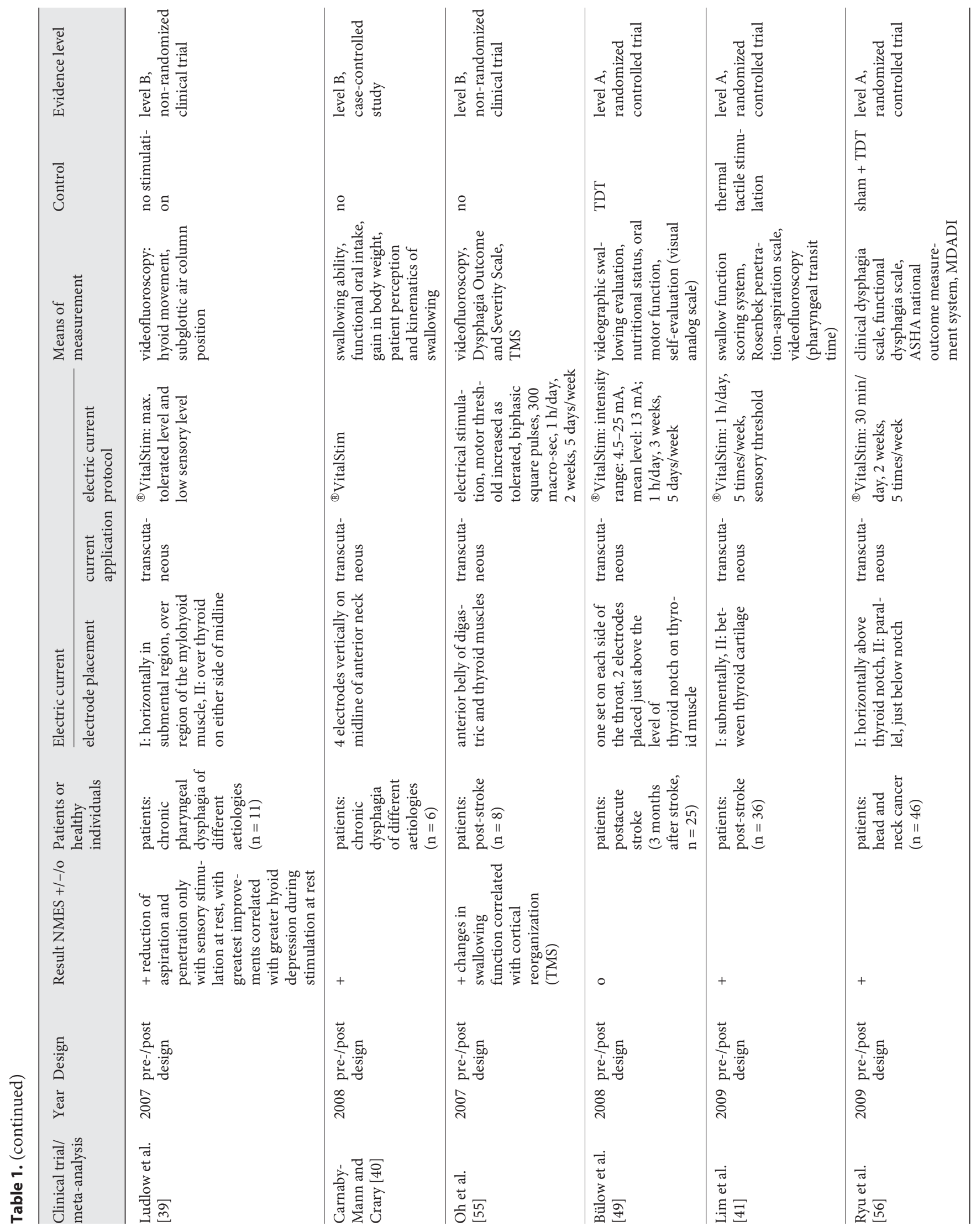




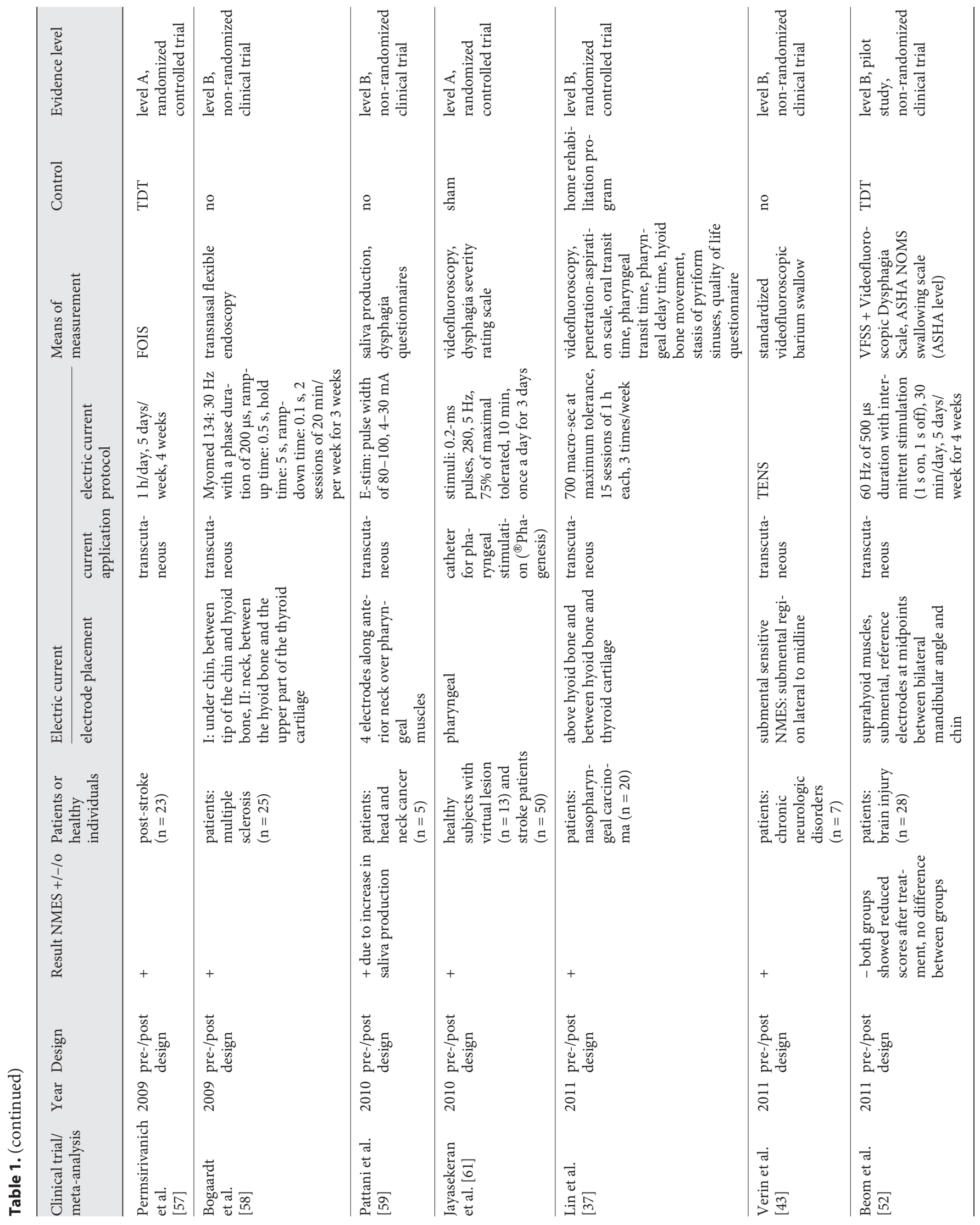




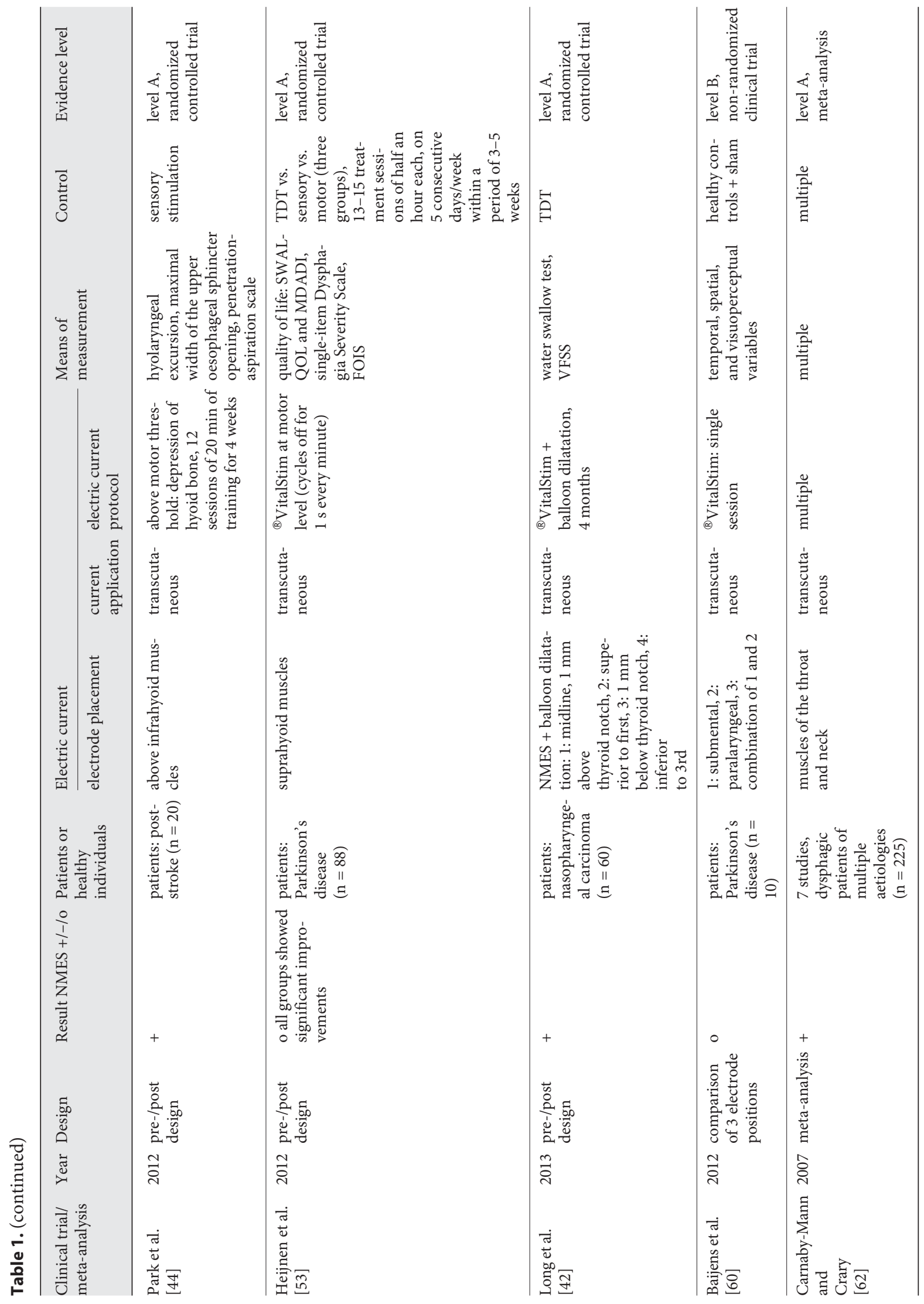



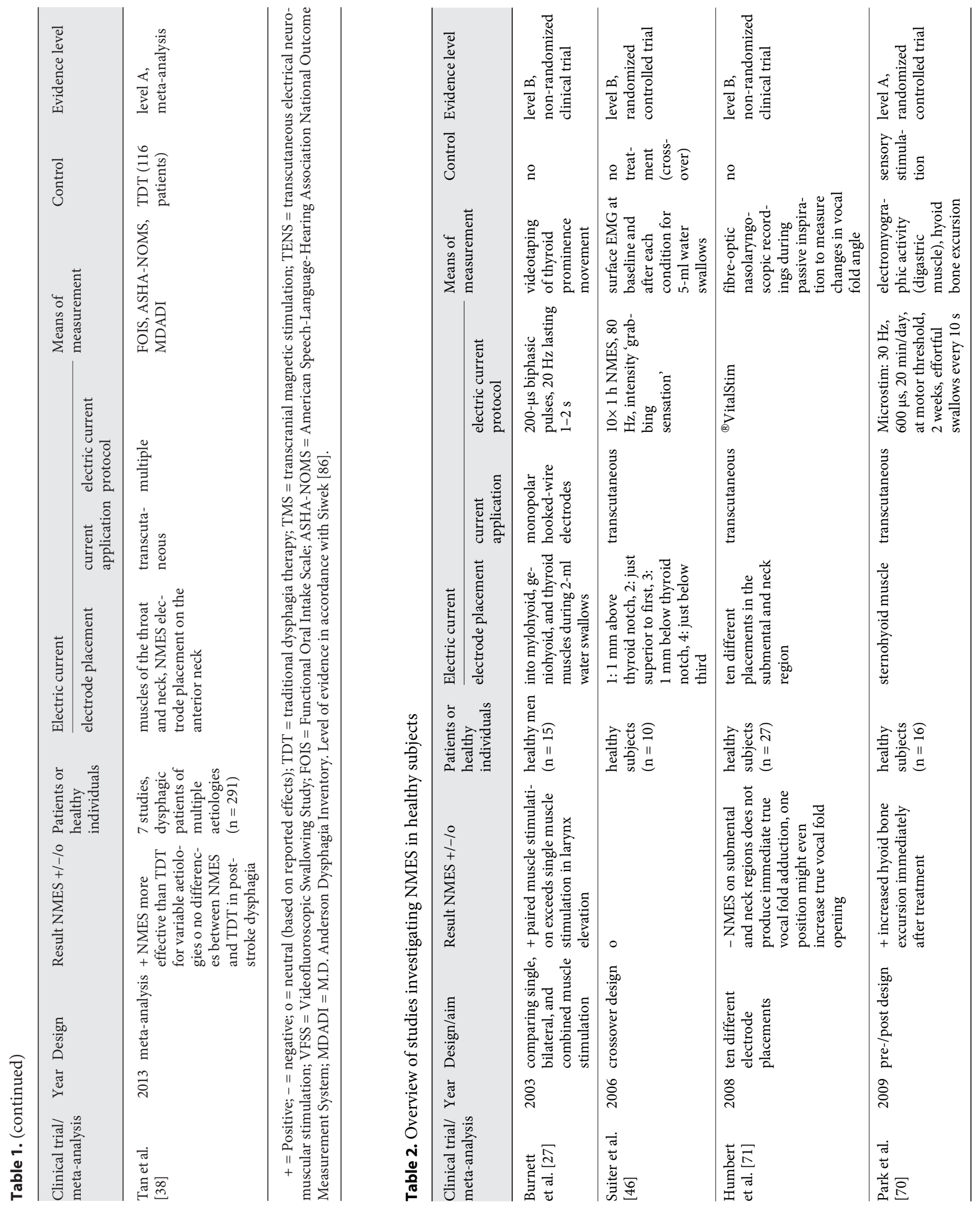


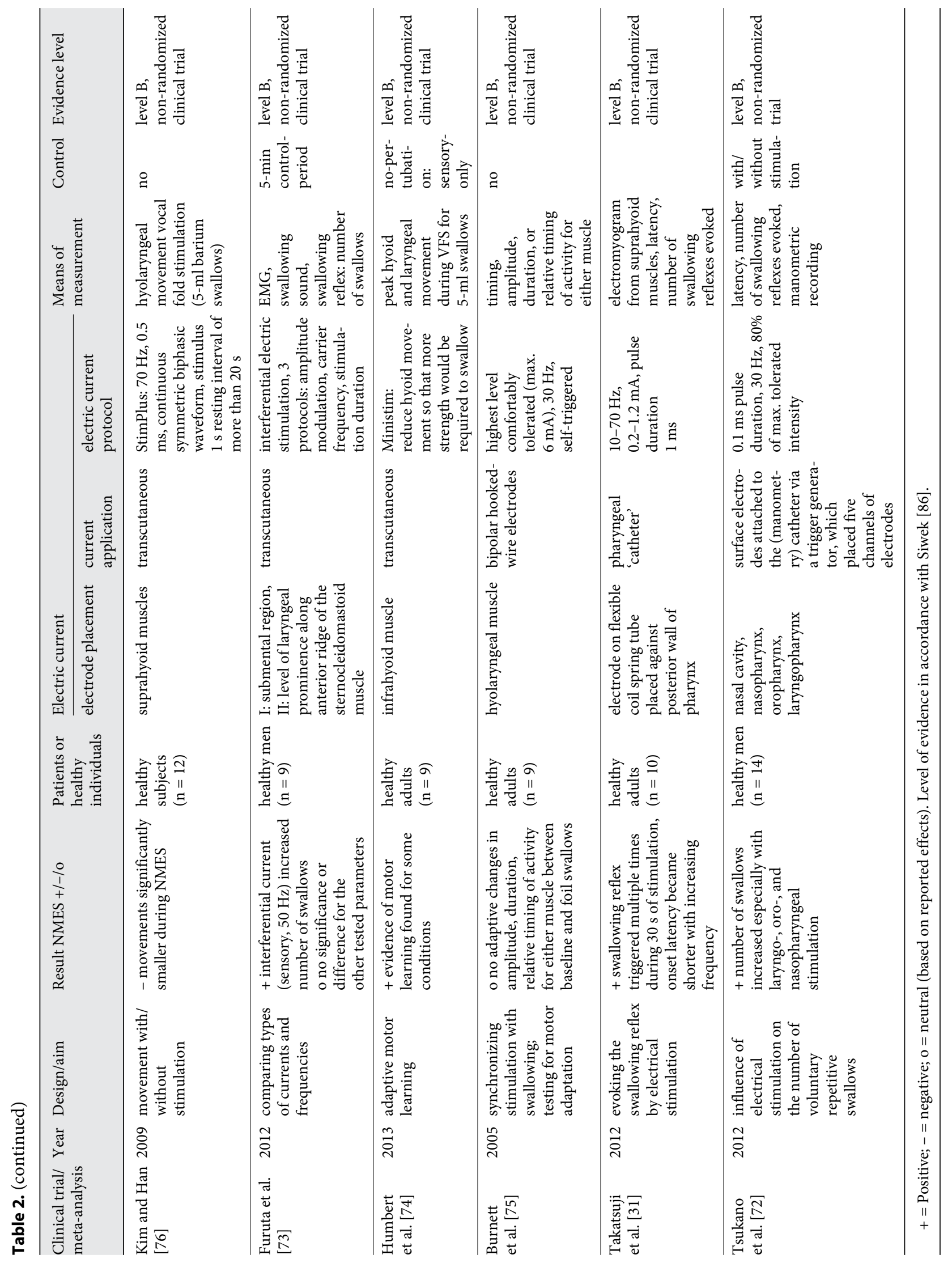


Whereas Suiter et al. [46] compared three different conditions of stimulation and found no significant differences comparing EMG measurements taken from the anterior neck $(1 \mathrm{~mm}$ superior to the thyroid notch and a second electrode positioned immediately superior to the first), other studies reported positive effects, such as Park et al. [70] who found that hyoid bone excursion showed an increase in their experimental group immediately after treatment. Also investigating hyoid movement, Burnett et al. [27] found that paired muscle stimulation exceeded individual/single muscle stimulation significantly. The results indicated also that no one muscle or muscle pair achieved the greatest elevation in all individuals, leading to the assumption that the extent of movement depended on the area of the muscle the stimulation had been applied to [27]. The theory of adaptive motor learning was investigated by Humbert et al. [74] as well as Burnett et al. [27] in order to investigate whether the hyolaryngeal movement adapts to the presence of a persistent perturbation resulting from the stimulation at motor level. Evidence of motor learning was found for a couple of conditions by Humbert et al. [74], but no significant changes were found by Burnett et al. [75].

Humbert et al. [71] compared ten different electrode placements and concluded that surface electrical stimulation to the submental and neck regions does not produce immediate true vocal fold adduction adequate for airway protection during swallowing. It was also put forward that NMES might even worsen swallowing activity by reducing swallow-related movements [71].

Turning to the question of different stimulation currents Furuta et al. [73] found that interferential current stimulation at the sensory threshold significantly increased the number of swallows and that pure alternating current stimulation failed to. The results led to the assumption that interferential current stimulation or lowfrequency, modulated kilohertz alternating current stimulation might be an alternative stimulation mode in the treatment of dysphagia.

Regarding swallowing frequency, the number of swallows increased significantly by applying NMES to the laryngo-, oro- and nasopharynx [73] and the onset latency of the swallowing reflex seemed to become shorter with increasing stimulation frequency (up to $30 \mathrm{~Hz}$ ) [72].

Ten studies [27, 31, 46, 70-76] were identified in this literature search. Six of these reported positive results in relation with NMES and swallowing (table 2).
NMES Studies regarding Cerebral Representation, Reorganization and Excitability

Longitudinal studies involving neurologic patients confirmed deglutition-related cortical changes and reorganization. Diseases in which there is degeneration of the upper and lower motoneuron showed decreasing cortical excitability [27, 31, 46, 70-76], whereas lesions beyond the upper motor neuron, e.g., bulbospinal muscle atrophy, typically result in an increase of the cortical representation of swallowing [77].

During the rehabilitation of stroke two processes might take place; either a restitution of impaired functions of the damaged hemisphere, or, more typically, functional reorganization processes of the unimpaired hemisphere, e.g. increase in motor representation of the pharynx [77].

Several studies investigated the effects exerted by NMES on the cerebral representation of swallowing [77, 78] (see table 3 for an overview). Some reported that input patterns associated with enhanced excitability (pharyngeal stimulation $5 \mathrm{~Hz}, 10 \mathrm{~min}$ ) induced stronger cortical activation [79-83]. When applied to acutely dysphagic stroke patients, corticobulbar excitability is increased mainly in the undamaged hemisphere, which correlates strongly with improvement in swallowing function [79]. Stimulating the pharynx at a frequency of $5 \mathrm{~Hz}$ is also said to result in increased cortical excitability and an expansion of the cortical area representing swallowing for up to $60 \mathrm{~min}$ following stimulation [79]. All of the identified studies [79-84] reported positive results on cortical excitability or reorganization in relation with NMES (table 3).

Effects of sensitive submental transcutaneous NMES were investigated by Fraser et al. [79, 80], Power et al. [81], Gallas et al. [82], Doeltgen et al. [83] and Hamdy et al. [84]. Even though results from Gallas et al. [82] suggested an improvement in the subjective swallowing rating and swallowing reaction time with a decrease in aspiration and residue, no modification of the motor pharyngeal cortical area was identified via transcranial magnetic stimulation. Doeltgen et al. [83], however, reported that $80-\mathrm{Hz}$ NMES increased the motor-evoked potential amplitude at 30 and 60 min after NMES, but only after a specific stimulation procedure. Similarly, Power et al. [81] reported that faucial pillar stimulation at $5 \mathrm{~Hz}$ decreased corticobulbar excitability and enhanced swallow response time, whereas stimulation at $0.2 \mathrm{~Hz}$ increased excitability without lengthening the response time. No modification could be found for $1 \mathrm{~Hz}$ and sham stimulation. 


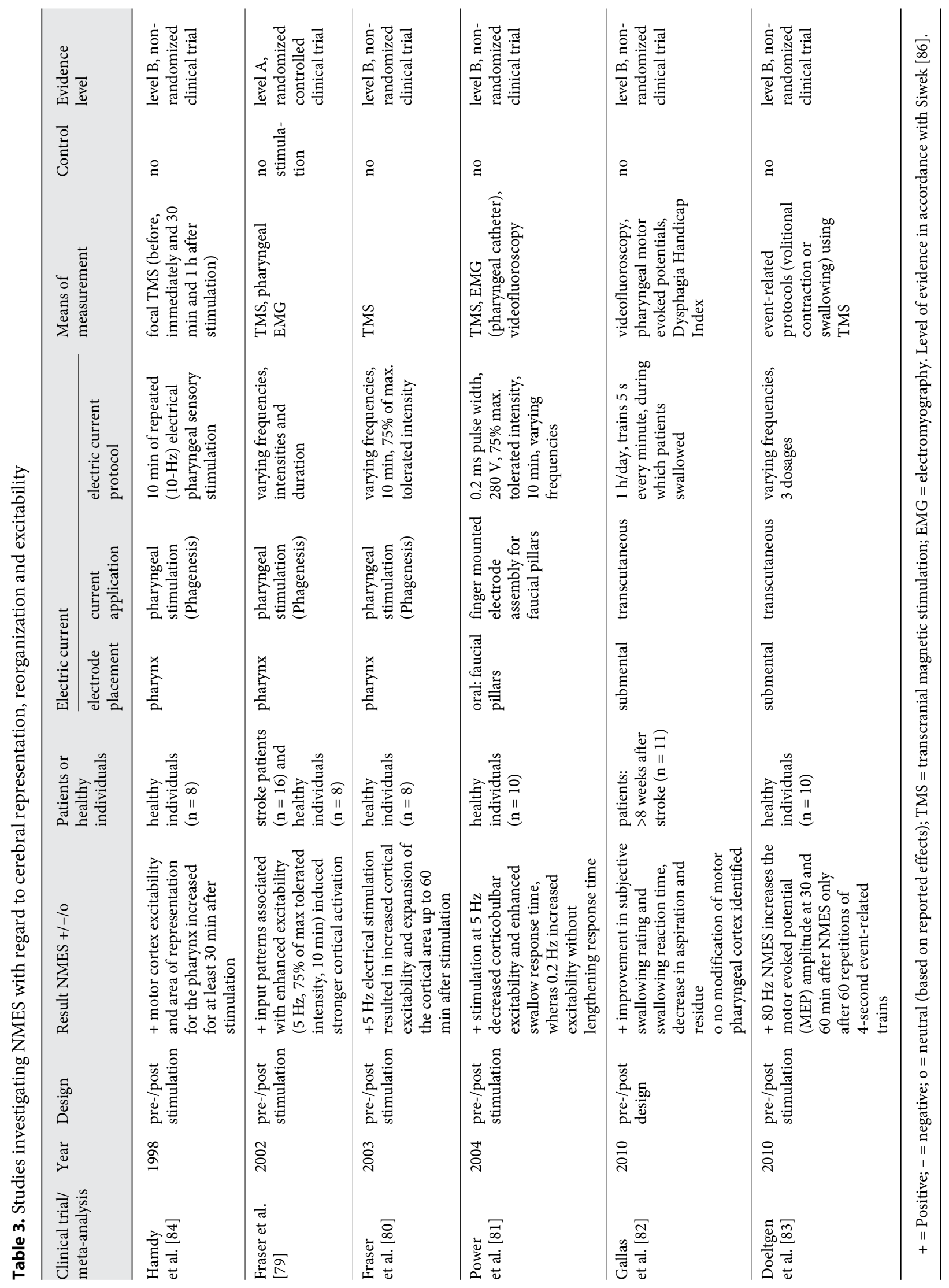




\section{Discussion}

\section{Limitations of This Paper}

Having only used PubMed as a search engine to identify studies in relation with NMES and dysphagia, dysphonia or dyspnoea further, possibly valuable studies may have been missed. Furthermore only free-text words were used, where the use of MESH terms might have identified more studies.

Due to length restrictions of this article not all of the identified studies are discussed in the text, where studies with exemplar results were chosen. Tables $1-3$ give an overview of the results.

\section{Conclusion}

Regarding basic science studies it appears reasonable to assume that NMES can modulate swallowing directly and/or by interfering with (central) control and execution mechanisms. In this case, NMES may offer hope for better therapeutic effects. Several clinical studies indicate a benefit of percutaneous and possibly invasive electrical stimulation approaches in the treatment of dysphonia. These findings are also very relevant for dysphagia therapy: they show that vocal fold closure is modifiable by NMES due to muscle weakness and even due to paresis.

Up to now, there have generally been three approaches in NMES for dysphagia. Oropharyngeal stimulation was applied via palatal prostheses which deliver electrical stimulation bilaterally to the faucial pillars, via electrodes mounted on a gloved finger or by an intraluminal pharyngeal catheter. Oropharyngeal stimulation has been shown to be relatively successful in modulating the swal- lowing reflex and corticobulbar excitability [81]. The application of transcutaneous stimulation is very difficult due to limited muscle specificity. A number of studies have been performed using this stimulation approach, but the effectiveness is still debated as a result of major methodological flaws in the study protocols $[79-81,85]$. Intramuscular stimulation, just as the previously mentioned approaches, has shown, some promising results [85]. Due to different stimulation protocols and the various underlying pathological conditions, a comparison of the current literature appears to be difficult. Furthermore spontaneous recovery may have occurred in patients treated with NMES.

There is also no consensus as to which type of current and parameters should be used, the exact timing of stimulation or electrode placement. Regarding the question whether NMES should be used in therapy for dysphagia and dysphonia, it can be concluded that considering the studies discussed, no side effects have been described. It can therefore be used in the therapy of laryngeal paresis, submental muscle support, enhancement of infrahyoidal resistance or to support sensory input. In combination with traditional therapy methods, NMES offers better and faster therapy effects. However, further well-implemented studies regarding the effectiveness and efficiency of NMES as a therapeutic approach in the treatment of dysphonia and dysphagia are needed.

\section{Disclosure Statement}

None declared.

\section{References}

1 Howes GJ: The phylogenetic relationships of the Electric Catfish Family Malapteruridae (Teleostei: Siluroidei). J Nat Hist 1985;19:3767.

2 Kane K, Taub A: A history of local electrical analgesia. Pain 1975;1:125-138.

3 Low J, Reed A: Electrical stimulation of nerve and muscle; in Low J, Reed A: Electrotherapy Explained: Principles and Practice, ed 3. Oxford, Buttlerworth-Heinemann, 2000, pp 53140.

4 Seidl RO, Nahrstaedt H, Schauer T: Electric stimulation in dysphagia therapy - a review. Laryngorhinootologie 2009;88:768-774.

5 Grill WM, Craggs MD, Foreman RD, et al: Emerging clinical applications of electrical stimulation: opportunities for restoration of function. J Rehabil Res Dev 2001;38:641-653.
6 Schönweiler R, Ptok M: Phoniatrie und Pädaudiologie. Krankheiten der Sprache, der Stimme und des Gehörs, ed 3. Lübeck, 2004.

7 Böhme G: The efficacy of electrotherapy in laryngeal diseases as shown in the stroboscopic picture. Z Laryngol Rhinol Otol 1965;44:481488.

8 Iakovleva II, Iliutovich GM, Iuvalova ND: On the use of sinusoidal low-frequency modulated currents in vocal disorders in patients with paralysis and paresis of the laryngeal muscles. Vestn Otorinolaringol 1965;27:9398.

-9 Pozsgay J, Heckenast O, Vincze L: Conservative management of recurrent nerve paralysis. HNO 1967;15:273-276.

10 Ptok M, Strack D: Voice exercise therapy versus electrostimulation therapy in patients with unilateral vocal fold paralysis. HNO 2005;53:1092-1097.

11 Ptok M, Strack D: Electrical stimulation-supported voice exercises are superior to voice exercise therapy alone in patients with unilateral recurrent laryngeal nerve paresis: results from a prospective, randomized clinical trial. Muscle Nerve 2008;38:1005-1011.

$\$ 12$ Zealear DL, Rainey CL, Herzon GD, et al: Electrical pacing of the paralyzed human larynx. Ann Otol Rhinol Laryngol 1996;105: 689-693.

13 Dahl R, Witt G: Analysis of voice parameters after conservative treatment of laryngeal paralysis with conventional voice Exercises or neuromuscular electrophonatory stimulation. Folia Phoniatr Logop 2006;58:415426. 
14 LaGorio LA, Carnaby-Mann GD, Crary MA: Treatment of vocal fold bowing using neuromuscular electrical stimulation. Arch Otolaryngol Head Neck Surg 2010;136:398-403.

15 Tanaka S, Hirano M, Chijiwa K: Some aspects of vocal fold bowing. Ann Otol Rhinol Laryngol 1994;103:357-362.

16 Thomas LB, Harrison AL, Stemple JC: Aging thyroarytenoid and limb skeletal muscle: lessons in contrast. J Voice 2008;22:430-450.

$\checkmark 17$ Ludlow CL, Schulz GM, Yamashita T, et al: Abnormalities in long latency responses to superior laryngeal nerve stimulation in adductor spasmodic dysphonia. Ann Otol Rhinol Laryngol 1995;104:928-935.

$\checkmark 18$ Bidus KA, Thomas GR, Ludlow CL: Effects of adductor muscle stimulation on speech in abductor spasmodic dysphonia. Laryngoscop 2000;110:1943-1949.

-19 Friedman M, Toriumi DM, Grybauskas V, et al: Treatment of spastic dysphonia without nerve section. Ann Otol Rhinol Laryngol 1987;96:590-596.

20 Hammer SS: Stimmtherapie mit Erwachsenen, ed 3. Heidelberg, Springer, 2007.

-21 Guirro RR, Bigaton DR, Silverio KC, et al: Transcutaneous electrical nerve stimulation in dysphonic women. Pro Fono 2008;20:189195.

22 Schnall RP, Pillar G, Kelsen SG, et al: Dilatory effects of upper airway muscle contraction induced by electrical stimulation in awake humans. J Appl Physiol 1995;78:1950-1956.

-23 Fowler LP, Gorham-Rowan M, Hapner ER: An exploratory study of voice change associated with healthy speakers after transcutaneous electrical stimulation to laryngeal muscles. J Voice 2011;25:54-61.

24 Bigenzahn W, Denk D: Oropharyngeale Dysphagien: Ätiologie, Klinik, Diagnostik und Therapie von Schluckstörungen. Stuttgart, Thieme, 1999.

25 Bartolome G: Grundlagen der funktionellen Dysphagietherapie (FDT); in Bartolome G, Schröter-Morasch H (Hrsg): Schluckstörungen. Diagnostik und Rehabilitation, ed 3. München, Elsevier, 2006, pp 245-360.

-26 Ludlow CL: Electrical neuromuscular stimulation in dysphagia: current status. Curr Opin Otolaryngol Head Neck Surg 2010;18:159164.

-27 Burnett TA, Mann EA, Cornell SA, et al: Laryngeal elevation achieved by neuromuscular stimulation at rest. J Appl Physiol 2003;94 128-134.

28 Hamdy S, Aziz Q, Rothwell JC, et al: Explaining oropharyngeal dysphagia after unilateral hemispheric stroke. Lancet 1997;350:686-692.

29 Leelamanit V, Limsakul C, Geater A: Synchronized electrical stimulation in treating pharyngeal dysphagia. Laryngoscope 2002; 112:2204-2210.

-30 Larsen GL: Conservative management for incomplete dysphagia paralytica. Arch Phys Med Rehabil 1973;54:180-185.

-31 Takatsuji H, Zakir HM, Mostafeezur RM, et al: Induction of the swallowing reflex by elec- trical stimulation of the posterior oropharyngeal region in awake humans. Dysphagia 2012, E-pub ahead of print.

-32 Blumenfeld L, Hahn Y, Lepage A, et al: Transcutaneous electrical stimulation versus traditional dysphagia therapy: a nonconcurrent cohort study. Otolaryngol Head Neck Surg 2006; 135:754-757.

33 Pahn J: Kurze Einführung in die Therapie von Larynxparesen, Aphasie, Dysphasie mit dem Gerätekonzept VocaSTIM. SchnaittachLaipersdorf, Physiomed Elektromedizin, 2002.

34 Freed ML, Freed L, Chatburn RL, et al: Electrical stimulation for swallowing disorders caused by stroke. Respir Care 2001;46:466474.

35 Chaudhuri G, Brady S, Caldwell R: Electric stimulation for dysphagia following stroke: pilot data. Arch Phys Med Rehabil 2006; 87:e51.

36 Huckabee ML, Doeltgen S: Emerging modalities in dysphagia rehabilitation: neuromuscular electrical stimulation. N Z Med J 2007; 120:U2744.

37 Lin PH, Hsiao TY, Chang YC, et al: Effects of functional electrical stimulation on dysphagia caused by radiation therapy in patients with nasopharyngeal carcinoma. Support Care Cancer 2011;19:91-99.

38 Tan C, Liu Y, Li W, et al: Transcutaneous neuromuscular electrical stimulation can improve swallowing function in patients with dysphagia caused by non-stroke diseases: a metaanalysis. J Oral Rehabil 2013;40:472-480.

- 39 Ludlow CL, Humbert I, Saxon K, et al: Effects of surface electrical stimulation both at rest and during swallowing in chronic pharyngeal dysphagia. Dysphagia 2007;22:1-10.

40 Carnaby-Mann GD, Crary MA: Adjunctive neuromuscular electrical stimulation for treatment-refractory dysphagia. Ann Otol Rhinol Laryngol 2008;117:279-287.

41 Lim KB, Lee HJ, Lim SS, et al: Neuromuscular electrical and thermal-tactile stimulation for dysphagia caused by stroke: a randomized controlled trial. J Rehabil Med 2009;41:174178.

42 Long YB, Wu XP: A randomized controlled trial of combination therapy of neuromuscular electrical stimulation and balloon dilatation in the treatment of radiation-induced dysphagia in nasopharyngeal carcinoma patients. Disabil Rehabil 2013;35:450-454.

43 Verin E, Maltete D, Ouahchi Y, et al: Submental sensitive transcutaneous electrical stimulation (SSTES) at home in neurogenic oropharyngeal dysphagia: a pilot study. Ann Phys Rehabil Med 2011;54:366-375.

44 Park JW, Kim Y, Oh JC, et al: Effortful swallowing training combined with electrical stimulation in post-stroke dysphagia: a randomized controlled study. Dysphagia 2012; 27:521-527.

45 Langdon C, Blacker D: Dysphagia in stroke: a new solution. Stroke Res Treat 2010;2010: 570403.
46 Suiter DM, Leder SB, Ruark JL: Effects of neuromuscular electrical stimulation on submental muscle activity. Dysphagia 2006;21: 56-60.

47 Humbert IA, Poletto CJ, Saxon KG, et al: The effect of surface electrical stimulation on hyolaryngeal movement in normal individuals at rest and during swallowing. J Appl Physiol 2006;101:1657-1663.

48 Kiger M, Brown CS, Watkins L: Dysphagia management: an analysis of patient outcomes using VitalStim therapy compared to traditional swallow therapy. Dysphagia 2006;21: 243-253.

49 Bülow M, Speyer R, Baijens L, et al: Neuromuscular electrical stimulation (NMES) in stroke patients with oral and pharyngeal dysfunction. Dysphagia 2008;23:302-309.

50 Christiaanse ME, Mabe B, Russell G, et al: Neuromuscular electrical stimulation is no more effective than usual care for the treatment of primary dysphagia in children. Pediatr Pulmonol 2011;46:559-565.

51 Power ML, Fraser CH, Hobson A, et al: Evaluating oral stimulation as a treatment for dysphagia after stroke. Dysphagia 2006;21:4955.

52 Beom J, Kim SJ, Han TR: Electrical stimulation of the suprahyoid muscles in brain-injured patients with dysphagia: a pilot study. Ann Rehabil Med 2011;35:322-327.

53 Heijnen BJ, Speyer R, Baijens LW, et al: Neuromuscular electrical stimulation versus traditional therapy in patients with Parkinson's disease and oropharyngeal dysphagia: effects on quality of life. Dysphagia 2012;27:336345

54 Shaw GY, Sechtem PR, Searl J, et al: Transcutaneous neuromuscular electrical stimulation (VitalStim) curative therapy for severe dysphagia: myth or reality? Ann Otol Rhinol Laryngol 2007;116:36-44.

55 Oh BM, Kim DY, Paik NJ: Recovery of swallowing function is accompanied by the expansion of the cortical map. Int J Neurosci 2007; 117:1215-1227.

56 Ryu JS, Kang JY, Park JY, et al: The effect of electrical stimulation therapy on dysphagia following treatment for head and neck cancer. Oral Oncol 2009;45:665-668.

57 Permsirivanich W, Tipchatyotin S, Wongchai $\mathrm{M}$, et al: Comparing the effects of rehabilitation swallowing therapy vs. neuromuscular electrical stimulation therapy among stroke patients with persistent pharyngeal dysphagia: a randomized controlled study. J Med Assoc Thai 2009;92:259-265.

58 Bogaardt H, van Dam D, Wever NM, et al: Use of neuromuscular electrostimulation in the treatment of dysphagia in patients with multiple sclerosis. Ann Otol Rhinol Laryngol 2009;118:241-246.

59 Pattani KM, McDuffie CM, Morgan M, et al: Electrical stimulation of post-irradiated head and neck squamous cell carcinoma to improve xerostomia. J La State Med Soc 2010; $162: 21-25$
Electrical Stimulation for

Pharyngolaryngeal Dysfunction
Folia Phoniatr Logop 2013;65:154-168 DOI: $10.1159 / 000355562$ 
60 Baijens LW, Speyer R, Passos VL, et al: The effect of surface electrical stimulation on swallowing in dysphagic Parkinson patients. Dysphagia 2012;27:528-537.

61 Jayasekeran V, Singh S, Tyrrell P, et al: Adjunctive functional pharyngeal electrical stimulation reverses swallowing disability after brain lesions. Gastroenterology 2010;138: 1737-1746.

62 Carnaby-Mann GD, Crary MA: Examining the evidence on neuromuscular electrical stimulation for swallowing: a meta-analysis. Arch Otolaryngol Head Neck Surg 2007;133: 564-571.

63 Clark H, Lazarus C, Arvedson J, et al: Evidence-based systematic review: effects of neuromuscular electrical stimulation on swallowing and neural activation. Am J Speech Lang Pathol 2009;18:361-375.

-64 Baijens LW, Speyer R, Roodenburg N, et al: The effects of neuromuscular electrical stimulation for dysphagia in opercular syndrome: a case study. Eur Arch Otorhinolaryngol 2008; 265:825-830.

65 Cheung SM, Chen CJ, Hsin YJ, et al: Effect of neuromuscular electrical stimulation in a patient with Sjögren's syndrome with dysphagia: a real time videofluoroscopic swallowing study. Chang Gung Med J 2010;33:338-345.

-66 Barikroo A, Lam PM: Comparing the effects of rehabilitation swallowing therapy vs. functional neuromuscular electrical stimulation therapy in an encephalitis patient: a case study. Dysphagia 2011;26:418-423.

67 Holmes SR, Gudridge TA, Gaudiani JL, et al: Dysphagia in severe anorexia nervosa: a case report. Int J Eat Disord 2012;45:463-466.
68 Holmes SR, Gudridge TA, Gaudiani JL, et al: Dysphagia in severe anorexia nervosa and potential therapeutic intervention: a case series. Ann Otol Rhinol Laryngol 2012;121:449-456.

69 Lee SY, Yang HE, Yang HS, et al: Neuromuscular electrical stimulation therapy for dysphagia caused by Wilson's disease. Ann Rehabil Med 2012;36:409-413.

70 Park JW, Oh JC, Lee HJ, et al: Effortful swallowing training coupled with electrical stimulation leads to an increase in hyoid elevation during swallowing. Dysphagia 2009;24:296301.

71 Humbert IA, Poletto CJ, Saxon KG, et al: The effect of surface electrical stimulation on vocal fold position. Laryngoscope 2008;118:14-19.

72 Tsukano H, Taniguchi H, Hori K, et al: Individual-dependent effects of pharyngeal electrical stimulation on swallowing in healthy humans. Physiol Behav 2012;106:218-223.

73 Furuta T, Takemura M, Tsujita J, et al: Interferential electric stimulation applied to the neck increases swallowing frequency. Dysphagia 2012;27:94-100.

74 Humbert IA, Christopherson H, Lokhande A, et al: Human hyolaryngeal movements show adaptive motor learning during swallowing. Dysphagia 2013;28:139-145.

75 Burnett TA, Mann EA, Stoklosa JB, et al: Selftriggered functional electrical stimulation during swallowing. J Neurophysiol 2005;94: 4011-4018.

76 Kim SJ, Han TR: Effect of surface electrical stimulation of suprahyoid muscles on hyolaryngeal movement. Neuromodulation 2009;12:134-140.

77 Teismann IK, Ringelstein EB, Dziewas R: Kortikale Repräsentation des Schluckaktes: Neues zur Physiologie und Pathophysiologie des Schluckens. Klin Neurophysiol 2009;40: 183-193.
78 Hamdy S, Aziz Q, Rothwell JC, et al: Recovery of swallowing after dysphagic stroke relates to functional reorganization in the intact motor cortex. Gastroenterology 1998;115:11041112.

79 Fraser C, Power M, Hamdy S, et al: Driving plasticity in human adult motor cortex is associated with improved motor function after brain injury. Neuron 2002;34:831-840.

$>80$ Fraser C, Rothwell J, Power M, et al: Differential changes in human pharyngoesophageal motor excitability induced by swallowing, pharyngeal stimulation, and anesthesia. Am J Physiol Gastrointest Liver Physiol 2003; 285:G137-G144.

81 Power M, Fraser C, Hobson A, et al: Changes in pharyngeal corticobulbar excitability and swallowing behavior after oral stimulation. Am J Physiol Gastrointest Liver Physiol 2004; 286:G45-G50.

82 Gallas S, Marie JP, Leroi AM, et al: Sensory transcutaneous electrical stimulation improves post-stroke dysphagic patients. Dysphagia 2010;25:291-297.

83 Doeltgen SH, Dalrymple-Alford J, Ridding MC, et al: Differential effects of neuromuscular electrical stimulation parameters on submental motor-evoked potentials. Neurorehabil Neural Repair 2010;24:519-527.

84 Hamdy S, Rothwell JC, Aziz Q, et al: Longterm reorganization of human motor cortex driven by short-term sensory stimulation. Nat Neurosci 1998;1:64-68.

85 Steele CM, Thrasher AT, Popovic MR: Electric stimulation approaches to the restoration and rehabilitation of swallowing: a review. Neurol Res 2007;29:9-15.

86 Siwek J, Gourlay ML, Slawson DC, et al: How to Write an Evidence-Based Clinical Review Article. Am Fam Physician 2002;65:251-258. 